\title{
Contents, Vol. 32, 1968
}

Condiderunt: A. v. ALBERTINI - A. GRUMBACH - H. MOOSER

Offizielles Organ der Schweiz. Mikrobiologischen Gesellschaft

Organe officiel de la Société Suisse de Microbiologie

Official Organ of the International Society of Geographical Pathology

REDACTORES J. LINDENMANN, Zurich J. R. RÜTTNER, Zurich

Vol. 32

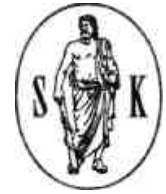

1968

BASEL (Schweiz)

S. KARGER

NEW YORK

Index

Agnihotri, V. P.: Studies on Colletotríchum capsicí. V. Utilization of Organic Acids . .

Ahmad-Zadeh, C.; Herzberg, M.; Paccaud, M. F. and Regamey, R. H.:

Immunological and Morphological Studies on Human Adenovirus

Type 31

254

Banic, S. und Budic, S.:

Vergleich des Einflusses von humanem und bovinem Serum auf die

Empfindlichkeit des Nährbodens B für die primäre Kultivierung von

Tuberkelbakterien 35

Barber, C; Eylan, E. et Keydar, J.:

Origine ribosomale des protéines communes des E. coli et des Salmo

nella 15

Bari, W. A.: Cysteine Protection Against the Morphologic Effects of X-Irradiation on Mouse Spleen 205

Ben-Ishay, Z.: vide Laufer, A.

Bertschinger, H. U.:

Zur Technik der serologischen Typisierung von E. coZ $Z_{i}$-Kul·turen aus

Schweinen mit Coli-Enterotoxaemie

91

Borman, J. B.: vide Laufer, A.

Bredt, W.: Motility and Multiplication of Mycoplasma pneumoniae. A Phase

Contrast Study 321

Budic, S.: vide Banic, S.

Chodat, F. et Elfiki, H.: 
Mutant achrome de Pseudomonas fluorescens et ses rapports avec la dissociation de cette espèce 86

Chuit, C. F.: Respiration dEscheríchía coli K12 resistant au chloramphénicol ou à la tétracycline 150

Corbaz, R.: Spores dans Tatmosphère 114

Cuppage, F. E. and Tate, A.:

Repair of the Nephron in Acute Renal Failure: Comparative Regene

ration Following Various Forms of Acute Tubular Injury 327

Davies, A. M.: vide Laufer, A.

Del Vecchio, V. G. and Turian, G.:

The Relationship of Ethanol Dehydrogenase to Morphogenesis of

Neurospora crassa 141

Dijk, A. van and Wikén, T. 0.:

On the Free Amino Acid Pool of Yeast Cells Showing a Negative

Pasteur Effect in Succinic Acid-Succinate Buffer

73

Dove, L. F.: vide Reuber, M. D.

Elekes, E.; Meréty, K. and Kocsár, L.:

The Action of Aluminium Hydroxide or Endotoxin on Natural Sheep

Haemolysin Producing Cells in Rats 345

Elfiki, H.: $\quad$ vide Chodat, F.

Emmrich, P. und Mälzer, G.:

Zur Morphologie der Plazenta bei Übertragung 285

Engelbrecht, E.: Sur une communauté antigénique entre Salmonella paratyphi B et

Salmonella bareílly 160

Engeler, M.-L.: vide Rüttner, J. R.

Ettlinger, L.: vide Glättli, $\mathrm{H}$.

Ettlinger, L.: vide Wyss, A.

Eylan, E.: $\quad$ vide Barber, C.

Fleurette, J. et Modjadedy, A.:

Etude critique des principaux caractères de pathogénicité de Staphylo

coccus aureus. Utilisation de la sérotypie comme méthode de compa-

raison 191

Foliguet, J. M.: vide Lavillaureix, J.

Galanti, B.; Paradisi, F.; Mancini, A. and Giusti, G.:

An Early Effect of Staphylococcus Alpha-Toxin on Cells Growing in

Culture: ATP Levels in Normal and Intoxicated Cells 277

Galanti, B.: vide Paradisi, F.

Gasser, M.: Immunität und Toleranz von Hamstern gegen heterologe Erythrozyten 137

Giusti, G.: $\quad$ vide Galanti, B.

Glättli, H. und Ettlinger, L.:

Äthanolverwertung durch Acetomonas oxydans

163

Glover, E. L.; Reuber, M. D. and Grollman, S.:

Influence of Age and Sex on Thyroiditis in Rats Injected Subcutane-

ously with 3-Methylcholanthrene 314

Glover, E. L.: vide Reuber, M. D.

Grieshaber, E.: vide Pedio, G. 
Grollman, S.: vide Glover, E. L.

Herzberg, M.: vide Ahmad-Zadeh, C.

Hottenrott, G.: vide Sprössig, M.

Hrabowska, M.: vide Kozlowski, H.

Keydar, J.: vide Barber, C.

Kocsár, L.: $\quad$ vide Elekes, E.

Kozlowski, H. and Hrabowska, M.:

Influence of Thioacetamide on the Evolution of Experimental Amy-

loidosis 228

Künzel, W. und Meissner, Ch. :

Über die Verwendung von /J-Propiolacton zur Herstellung von Myko-

plasmenmedien $\quad 353$

Laufer, A.; Ben-Ishay, Z.; Davies, A. M. and Borman, J. B.:

Electron Microscope Study of Auricular Biopsies in Chronic Heart

Disease $\quad 49$

Lavillaureix, J.; Schwarzbrod, L.; Foliguet, J. M. et Tinland, G.:

Etude postvaccinale d'une épidémie à polio-virus I

Löffler, H.: $\quad$ vide Meyer, J,

Mälzer, G.: $\quad$ vide Emmrich, P.

Mancini, A.: vide Galanti, B.

Mancini, A.: vide Paradisi, F.

Meissner, Ch. : vide Künzel, W.

Mendris, J. N.: vide Papaevangelou, G. J.

Merétey, K.: vide Elekes, E.

Meyer, J. und Löffler, H.:

Infektion gravider Mäuse mit Coxsackievirus

Modjadedy, A.: vide Fleurette, J.

Münter, W.: Kapseltypen und Antibiotika-Resistenz von Kie $\delta \mathrm{s}_{¿} \mathrm{e} /{ }_{\complement} \mathrm{o}-\mathrm{Stämmen}$ aus

klinischem Untersuchungsmaterial 305

Nicolet, J.: Un problème d'actualité: les mycotoxicoses 124

Paccaud, M. F.: vide Ahmad-Zadeh, C.

Papaevangelou, G. J. and Mendris, J. N.:

Evaluation of the Haemagglutination Inhibition Test for the Sero-

lógic Diagnosis of Rubella 267

Pakadisi, F.; Galanti, B. and Mancini, A.:

ATP Levels in Normal and Diphtheria Toxin Treated Cell Cultures 24

Paradisi, F.: vide Galanti, B.

Pedio, G.; Grieshaber, E. and Rüttner, J. R.:

On Plasmocytoma-Oncogenesis of Mice. II. Differential Ultracentri-

fugate which Contains RNA Virus-like Particles from HIPA Tumor

Inducing a Plasmocytoid Neoplasia in BALB/c Mice 241

Regamey, R. H.: vide Ahmad-Zadeh, C.

Reuber, M. D.; Dove, L. F. and Glover, E. L.:

Severe Cirrhosis with Hepatic Vein Thrombosis in Rats Given Carbon

Tetrachloride and 3-Methylcholanthrene

41

Reuber, M. D.: vide Glover, E. L. 
Rüttner, J. R.; Spycher, M. A. and Engeler, M.-L.:

Pulmonary Fibrosis Induced by Cotton Fibre Inhalation

Rüttner, J. R.: vide Pedio, G.

Schwarzbhod, L.: vide Lavillaureix, J.

Sprössig, M. und Hottenrott, G.:

Fluoreszenzserologische Studien bei letal verlaufenen Adenoviruspneu-

mopathien 219

Spycher, M. A.: vide Rüttner, J. R.

Tate, A.: $\quad$ vide Cuppage, F. E.

Tinland, G.: vide Lavillaureix, J.

Turian, G.: $\quad$ Sporulation fongique 98

Turian, G.: $\quad$ vide Del Vecchio, V. G.

Wikén, T. O.: vide Dijk, A. van

Wood, M.: Aetiology of Tumors of the Urinary Bladder. Induction of Tumors in

the Urinary Tract of the Rabbit by Aromatic Amines

177

Wyss, A. und Ettlinger, L.:

Über ein Essigsäurebakterium, das Essigsäure oxydieren, aber nicht

als einzige Kohlensto£fquelle gebrauchen kann 169

Book Reviews - Buchbesprechungen - Livres Nouveaux ... 60, 175, 237, 303, 360

Selman A. Waksman zum 80. Geburtstag

58

Proceedings - Verhandlungsberichte - Congrès

Société Suisse de Microbiologie, 27e assemblée annuelle, le 14 et 15 juin 1968 à Neuchâtel -

Schweizerische Mikrobiologische Gesellschaft, 27. Jahresversammlung, 14. und 15.Juni 1968 in Neuenburg .... 65

Coming Medical Meetings - Kongresskalender - Tableau des Congrès $\quad 240$

Varia 367

All rights, including those of translation into other languages, reserved.

Photomechanical reproduction (photocopy, microcopy) of this volume or parts thereof without special permission of the publishers is prohibited.

(C)

Copyright 1968 by S. Karger AG., Basel.

Printed in Switzerland by Friedrich Reinhardt AG, Basel

Blocks: Aberegg-Steiner \& Co., Bern, and Sterner \& Co., Basel 